\title{
COMPARACIÓN DE ANOMALÍAS DE GRAVEDAD DERIVADAS DE ALTIMETRÍA SATELITAL CON DATOS DE GRAVEDAD MARINA EN EL MARGEN CONTINENTAL ARGENTINO
}

Comparison of gravity anomalies from satellite altimetry with marine gravity data in the Argentine continental margin

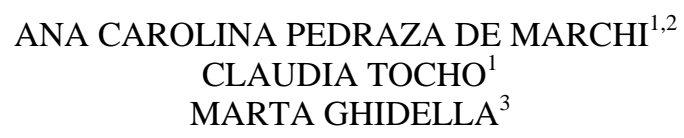

${ }^{1}$ Departamento de Gravimetría

Facultad de Ciencias Astronómicas y Geofísicas (FCAGLP)

Paseo del Bosque s/n, 1900 La Plata, Argentina

${ }^{2}$ Consejo Nacional de Investigaciones Científicas y Técnicas (CONICET)

Argentina.

${ }^{3}$ Instituto Antártico Argentino (IAA)

Cerrito 1248, 1010 Buenos Aires, Argentina.

cpedrazadm@fcaglp.unlp.edu.ar; ctocho@fcaglp.unlp.edu.ar; mghidella@dna.gov.ar

\section{RESUMEN}

En este trabajo se comparan grillas de anomalías de gravedad derivadas de misiones de altimetría satelital con anomalías de gravedad marina a partir de datos de cruceros en el margen continental argentino y zona oceánica adyacente. Se analizan las diferencias entre estas fuentes de datos, ya que es de esperar que éstas sean significativas en zonas costeras o de gradiente horizontal de gravedad abrupto como en el talud continental; se trata de identificar y delimitar dichas zonas. Asimismo se analizan los datos de barco de distintas campañas realizadas en épocas diferentes para organizarlos en una base de datos común, como parte de un proceso de compilación en el que se crearán versiones mejoradas con nuevas correcciones en la medida en que se incorporen más datos en el futuro. Se investiga cuál de las grillas de gravedad derivada de altimetría satelital presenta mejor ajuste con la compilación marina a fin de caracterizarla como la recomendable para su uso en posteriores 
aplicaciones. Se presentan aquí los resultados de la primera etapa completada en función del alcance los objetivos mencionados.

Palabras clave: Altimetría Satelital; Gravedad Marina;Bbase de Datos; Margen Continental Argentino.

In this paper we address the comparison among grids from different altimetryderived global gravity anomaly datasets with marine gravity anomalies from cruises in the Argentinian continental margin and adjacent oceanic areas in order to determine if there are significant differences among these data sources, particularly in areas near the coast where there are steep horizontal gravity gradients as over the continental slope, and to identify and delimit those areas. We also analyze the information of ship-borne data from different campaigns carried out at different times and organize them in a consistent database, in a compilation process in which improved versions, new corrections have been created. We investigate which satellite altimetry-derived gravity anomaly grid depicts the optimal fit to the marine data compilation in order to find the one to recommend for use in subsequent applications. The results here presented are the first completed series due to the scope of these reached goal.

Keywords: Satellite Altimetry; Marine Gravity; Data Base; Argentine Continental Margin.

\section{INTRODUCCIÓN}

En distintas décadas se han realizado alrededor del mundo mediciones de gravedad marina desde plataformas a bordo de barcos. Debido a errores del instrumental, de navegación y otras fuentes de error, existen diferencias significativas entre las mediciones de gravedad realizadas en distintos cruceros. La exactitud de los datos de barco está limitada principalmente por el error de navegación que afecta el cálculo del efecto Eötvös (DENKER y ROLAND, 2003). En contraste con las anomalías de gravedad medidas a bordo de barcos, las anomalías de gravedad derivadas de multimisiones satelitales tienen una cobertura homogénea y global, disponibles en grillas de dominio público, las que han sido calculadas, salvo algunas diferencias, usando la misma combinación de datos y distintas filosofías computacionales de cálculo (FEATHERSTONE, 2003). Para resolver cuál de estas grillas es superior en precisión o exactitud o es más representativa de los datos de la región del margen continental argentino, se plantea la comparación con las anomalías de gravedad marina obtenidas desde gravímetros a bordo de barcos. Se dispone de datos de las bases de datos del Bureau Gravimétrique Internacional (BGI) y de las campañas BGR87, BGR98 (1 y 2) realizadas por el Federal Institute for Geosciences and Natural Resources. En el presente trabajo se comparan cuatro grillas recientes de anomalías de gravedad derivadas de altimetría satelital en el margen continental argentino y zona oceánica 
adyacente llamadas DNSC08GRA (ANDERSEN et al., 2009), DTU10GRA (ANDERSEN, 2010) y las versiones V16.1 y V18.1 de Sandwell y Smith (SANDWELL y SMITH, 1997; SANDWELL y SMITH, 2009).

Como existen diferencias entre las distintas fuentes de datos, para decidir sobre la exactitud y precisión de las anomalías derivadas de altimetría satelital respecto a las anomalías de gravedad de barco hace falta contar con que estas últimas constituyen una fuente de datos de mayor precisión, para lo cual esta base de datos debe ser consistente y documentada. En una base consistente los datos han sido cuidadosamente editados, se han eliminado datos ruidosos propios del sistema de registración influenciados por los movimientos de rotación del barco y se les ha hecho algún tipo de corrección por cruces (cross-overs), primero para evaluar el acuerdo interno de los datos y segundo entre diferentes campañas para evaluar el acuerdo externo.

Inicialmente se utilizan los datos de altimetría satelital para descartar líneas de barco muy ruidosas o con algún error sistemático que produce diferencias muy grandes respecto a los datos derivados de altimetría, en los que para cada juego de comparación se tiene la estadística de las trazas individualizadas, lo que posibilita identificar aquellas que producen las mayores diferencias; al visualizar gráficamente dichas trazas puede identificarse la mala calidad del dato ya sea por falta de continuidad en la registración o por la alta variabilidad del mismo (posible ruido).

Como primer paso en el proceso de compilación, se comienza con la edición manual línea por línea de los datos de barco de cada campaña, y luego se hace la comparación nuevamente con los datos derivados de altimetría.

\section{ANOMALÍAS DE GRAVEDAD DERIVADAS DE ALTIMETRÍA}

Para calcular anomalías de gravedad a partir de altimetría desde satélite algunos autores usan técnicas que incluyen: 1) la conversión de la altura del geoide marino (corregido por la topografía de la superficie del mar) usando la fórmula inversa de Stokes en el dominio espectral vía FFT (ANDERSEN et al., 1998), y 2) la conversión de la deflexión de la vertical vía integración de la ecuación de Laplace (SANDWELL y SMITH, 1997). En estos métodos se usa la técnica remove-restore, que hace uso de un modelo geopotencial durante la conversión de la deflexión de la vertical a anomalías/perturbación basado en la transformada rápida de Fourier (FFT). En el uso de FFT existe un problema asociado con el efecto de borde dado que no se tienen datos altimétricos en tierra, y también resulta más problemático el grillado de los datos. Además, distintos grupos tienden a usar algoritmos diferentes de grillado de los datos como splines o mínimos cuadrados por colocación, por lo que se esperan diferencias entre grillas de anomalías de gravedad debido exclusivamente a la técnica usada (FEATHERSTONE, 2003). 


\section{DATOS UTILIZADOS}

\subsection{Grillas de Anomalías de Aire Libre Derivadas de Altimetría Satelital}

\subsubsection{DNSC08GRA}

Las anomalías de gravedad se derivan de la ondulación del geoide usando técnicas de FFT en el dominio de la frecuencia. Se incluye un filtro tipo Wiener para suavizar el campo de gravedad, que toma el valor 0.5 para la frecuencia de corte determinada empíricamente; longitudes de onda menores están dominadas por ruido y causan el llamado "efecto de piel de naranja" de los campos de gravedad derivados de altimetría (ANDERSEN et al., 1998),. El filtro fue diseñado usando 10 años de observaciones de ERS-2 en las que las regiones de alta variabilidad de la altura de la superficie del mar se filtraron más que las de regiones calmas. Luego de aplicar el filtro se restaura la contribución de las anomalías de gravedad del modelo EGM2008 (PAVLIS et al., 2008). La mejora en precisión y exactitud de este modelo en general sigue los mismos pasos que la versión V18.1 de Sandwell. Se aplicó una técnica nueva de análisis descendente y ascendente de traza (doubleretracking) a los datos de ERS-1, la que difiere de las aplicadas por Sandwell (SANDWELL y SMITH, 1997) dado que incluye un paso adicional de tolerancia múltiple adaptiva a las condiciones locales. Este paso adicional cubre de un 6 a $9 \%$ más datos en regiones de aguas someras y regiones polares.

En altas latitudes se incluyen datos de altimetría de ICESAT (Ice, Cloud, and land Elevation Satellite) y datos marinos en el océano Ártico del proyecto de gravedad del Ártico (ArcGP) que dan exactitud y mayor cobertura al campo de gravedad. Usando estos datos es posible alcanzar las regiones polares de $82^{\circ} \mathrm{N}$ donde no se tienen datos de altimetría previa.

Se usa el nuevo modelo geopotencial global EGM2008 más preciso en seis veces al EGM96 (Lemoine et al., 1998). Esto da más exactitud en el proceso remove-restore que con cualquier otra señal geopotencial. Se utiliza un modelo de topografía dinámica media DOY07A junto al EGM2008 para corregir a la altura de la superficie del mar. Este modelo está disponible en http://www.space.dtu / con resolución 1 minuto de arco.

DTU10GRA es el campo de gravedad global sucesor del DNSC08GRA. Tiene mejoras en la edición, retracking en ERS-1 GM, contempla información del cambio climático (retiro de hielos) y aunque aún falta mejorar el rango y correcciones geofísicas (marea oceánica, etc.) e implementar una corrección anual con datos estacionales, representa una mejora respecto del DNSC08GRA ya que incluye retracking en ERS-2 y en ENVISAT (ANDERSEN, 2010).

\subsubsection{V16.1 y V18.1}

Son las series más recientes de anomalía de gravedad marina de Sandwell, (SANDWELL y SMITH, 2009) de resolución 1 minuto de arco. 
Se utilizaron perfiles de altimetría satelital de las misiones Geosat Geodetic y de ERS-1 que se convirtieron a grillas de gradiente vertical de gravedad y anomalía de gravedad.

Las anomalías de gravedad se calcularon utilizando FFT inversa en la ecuación de Laplace con deflexión de la vertical, usando un modelo de referencia en la implementación de la FFT y el método remove-restore: el campo de referencia es removido de la deflexión de la vertical y es restaurado en la conversión a anomalías de gravedad (Sandwell y Smith, 1997). Se han utilizado los modelos EGM96 para la versión V16.1 y EGM2008 para la V18.1.

En la versión V18.1 se aplicaron mejoras vinculadas al reproceso de datos de ERS-1 y GEOSAT/GM, empleando retracking en datos de 11 meses de ERS-1 y 18 meses Geosat/GM, dando un rango de precisión de un 40\% y 27\% respectivamente (SANDWELL y SMITH, 2009). Se usó el nuevo modelo geopotencial EGM2008 en el método de remove-restaure, obteniéndose así una resolución de anomalías de gravedad de 5 minutos sobre tierra (EGM2008) y 1 minuto sobre los océanos, otorgando entonces continuidad a la transición tierra-océano. Se usó interpolación bi-armónica en la construcción de grillas de deflexión de la vertical residual. Se incluyó un modelo de la topografía dinámica media (MDOT) junto al EGM2008 para corregir una porción de las pendientes de la superficie del mar asociada a corrientes limítrofes occidentales resultando esto en una mejora de 6-10 mGal en la exactitud de las anomalías de gravedad en esas áreas. Se puede disponer de estas grillas en ftp://topex.ucsd.edu/pub/global_grav_1min/.

\subsection{Datos de Barco en El Margen Continental Argentino}

Por acuerdos de cooperación existentes se tiene acceso a dos conjuntos de datos de dos campañas marinas llevadas a cabo por el BGR, Bundenstalt für Geowissenshaften and Rohstoffe (Federal Institute for Geosciences and Natural Resources). Se dispone también de los informes técnicos de los cruceros, que fueron hechos en 1987 (BGR87) y en 1998 (BGR98), respectivamente. De dichos informes se sabe que para BGR87 se usó un gravímetro marino Bodenseewerk modelo KSS $\mathrm{N}^{\mathrm{o}}$ de serie 30. El crucero BGR98 fue desarrollado en dos etapas, diciembre de 1998 (BGR98-1) y enero de 1999 (BGR98-2) con un gravímetro marino LaCoste \& Romberg modelo S, de tipo aire-mar, № de serie S-105. El sistema de referencia utilizado para los datos gravimétricos fue el I.G.N.S 71 (International Gravity Standardization Net 1971). Se les ha aplicado la corrección de Eötvös con los datos de navegación, corrección por drift instrumental y substracción de la gravedad normal WGS 67 (World Geodetic Sistem 1967). La navegación se apoyó en el sistema de posicionamiento GPS (Global Positioning System), y el sistema de satellite de navegación (Transit Satellite System o Navy Navigation Satellite System, NNSS).

Otro conjunto de datos utilizados es el BGI, que es una compilación de varios años de toma de medidas (1941-1988), las cuales fueron registradas por el Bureau Gravimetrique International en los que entre otros datos se incluyen el número de 
fuente, coordenadas (latitud y longitud), gravedad observada en microGal, anomalía de aire libre, anomalía de gravedad de Bouguer, estimación de los errores en las anomalías, y un código que representa el instrumento utilizado entre otros datos.

Se tiene un número de 7537 observaciones de BGI, 10465 de BGR87, 95566 de BGR98-1 y 76086 de BGR98-2 en un área comprendida entre las latitudes $36^{\circ} \mathrm{y}$ $48^{\circ}$ Sur y las longitudes $62^{\circ}$ y $48^{\circ}$ Oeste.

Las anomalías de aire libre medidas por las campañas de barco están referidas a GRS67 (Sistema de Referencia Geodésico 1967). Para referirlas al GRS80 (Sistema de Referencia Geodésico 1980) utilizamos la siguiente fórmula:

$$
\Delta g_{\text {GRS } 80}=\Delta g_{\text {GRS } 67}+\gamma_{\text {GRS } 67}-\gamma_{\text {GRS } 80}
$$

donde $\Delta g_{\text {GRS80 }}$ y $\Delta g_{\text {GRS67 }}$ son las anomalías de gravedad en el sistema GRS80 y GRS67, respectivamente, y $\gamma_{\text {GRS80 }}$ y $\gamma_{\text {GRS67 }}$ los valores de gravedad normal en los sistemas GRS80 y GRS67, respectivamente.

$$
\begin{aligned}
& \gamma_{\text {GRS } 67}=978031 \quad .8\left[1+0.0053024 \quad \operatorname{sen}^{2}(\varphi)-0.0000059 \quad \operatorname{sen}^{2}(2 \varphi)\right] \mathrm{mGal} \\
& \gamma_{\text {GRS 80 }}=978032.7\left[1+0.0053024 \operatorname{sen}^{2}(\varphi)-0.0000058 \operatorname{sen}^{2}(2 \varphi)\right] \mathrm{mGal}
\end{aligned}
$$

en las que $\varphi$ es la latitud geodésica, 978031.8 es el valor de gravedad normal en el ecuador para el sistema GRS67 y 978032.7 para el sistema GRS80.

Estos datos no están aun editados ni corregidos por cruces por lo que no pueden proveer un control definitivo de la exactitud de las grillas de altimetría satelital en la región del margen continental argentino. La comparación directa de las diferentes fuentes de datos podrá mostrar niveles de acuerdo (quizás precisión) pero no exactitud.

En las Figuras 1 y 2 se puede observar la distribución de las trazas de cada campaña gravimétrica: en la Figura 1a las de BGR98 en su etapa 1 (BGR98-1), en la Figura 1b las de BGR98 su etapa 2 (BGR98-2), en la Figura 2a las de BGR87 y en la Figura 2b las de BGI. 
Figura 1: Derrotas de barco de BGR98-1(a), izquierda y BGR98-2(b), derecha.

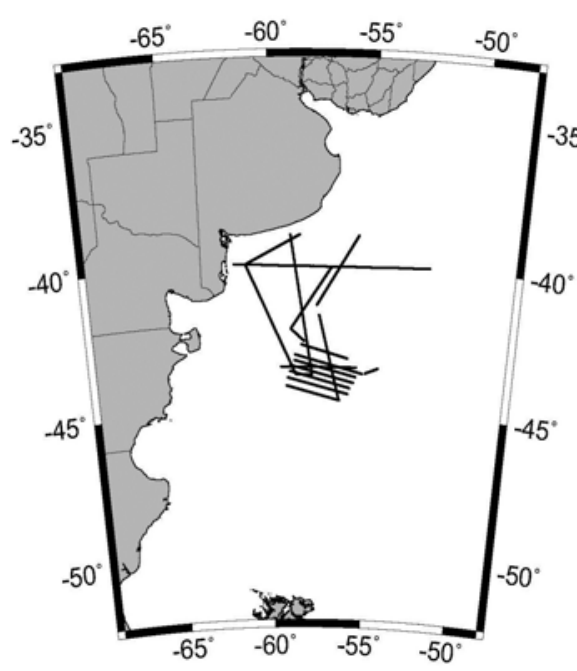

(a)

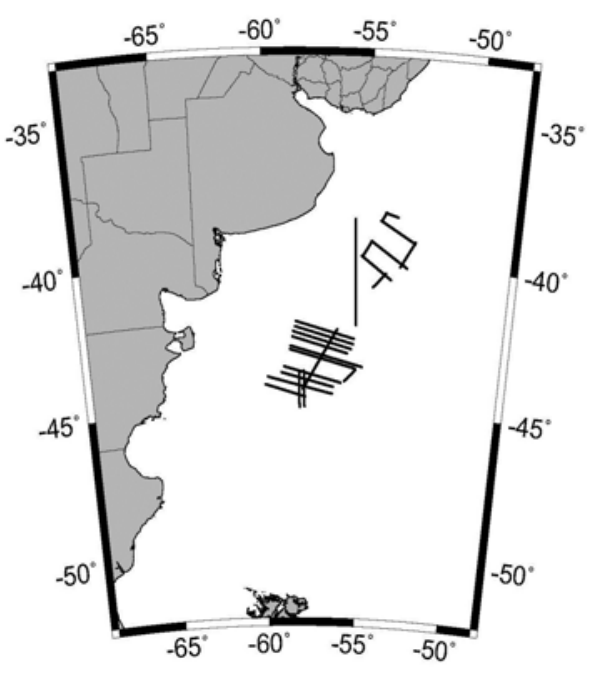

(b)

Figura 2: Derrotas de barco de BGR87(a), izquierda y BGI (b), derecha.

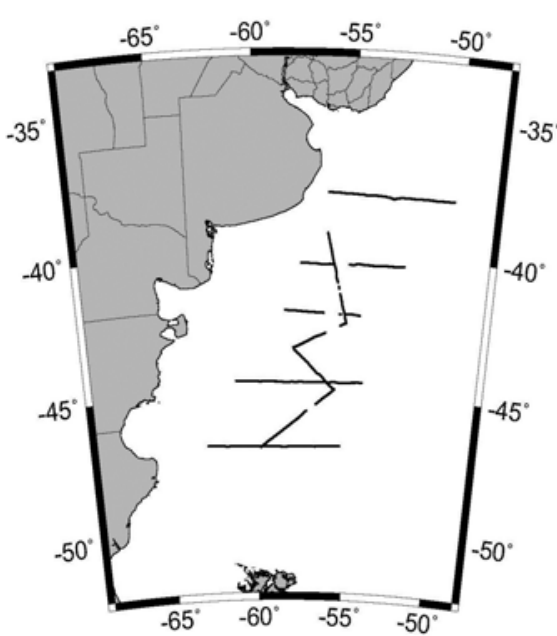

(a)

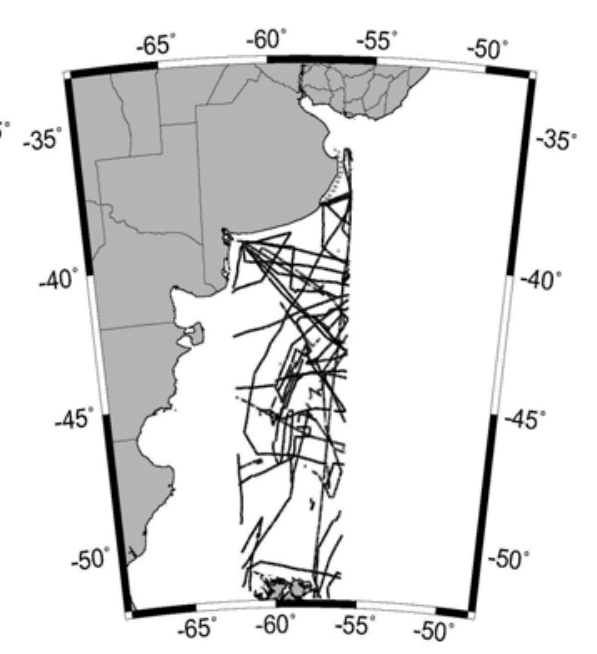

(b)

Bol. Ciênc. Geod., sec. Artigos, Curitiba, v. 18, nº 1, p.22-39 jan-mar, 2012. 


\section{COMPARACION Y DISCUSION}

\subsection{Comparación entre grillas de anomalías de gravedad derivadas de} altimetría satelital

Se extrajeron grillas rectangulares de bases de datos altimétricas en el área de estudio, a las que se hace referencia en el texto con los nombres SyS16.1 (V16.1), SyS18.1 (V18.1), DNSC08 (DNSC08GRA) y DTU10 (DTU10GRA).

Como las anomalías de gravedad hacen uso del modelo EGM96 (V16.1) o EGM2008 (V18.1, DNSC08GRA Y DTU10GRA) en tierra, en cada grilla se remueve esta contribución usando la rutina grdlandmask de GMT 4.5.3 (WESSEL y SMITH, 2007). Con este programa se puede generar una grilla auxiliar en la que los nodos en tierra emergida (continentes e islas) son remplazados por NAN (identificador de ausencia de datos) y los nodos en mar toman el valor 1. Esto constituye una máscara que puede utilizarse como un filtro espacial para eliminar los datos de tierra con la rutina grdmath, quitando así su influencia antes del cálculo de la estadística.

En la Tabla 1 se muestra la estadística de las grillas de anomalías de gravedad derivadas de altimetría satelital, en el área de los mapas de la Figura 3. El símbolo $\sigma$ representa la desviación estándar. Puede verse que en la Tabla 1 hay un buen acuerdo de unas a otras lo que es de esperar dado el uso de los mismos datos.

Tabla 1: Estadística descriptiva de las anomalías de gravedad derivadas de altimetría. Unidad: [mGal].

\begin{tabular}{cccccc}
\hline Grilla & $\mathrm{N}^{\circ}$ de puntos & Máximo & Mínimo & Media & $\sigma$ \\
SyS16.1 & 1011676 & 84.60 & -105.80 & -3.07 & 19.83 \\
SyS18.1 & 1011676 & 95.13 & -103.83 & -3.32 & 19.45 \\
DNSC08 & 1011676 & 95.35 & -102.94 & -3.27 & 19.46 \\
DTU10 & 1011676 & 95.52 & -102.51 & -3.28 & 19.40 \\
\hline
\end{tabular}

Tanto V16.1 como V18.1, DNSC08GRA y DTU10GRA son grillas de 1 minuto de arco por 1 minuto de arco; de ellas se extrajeron datos para la región en las grillas SyS16.1, SyS18.1, DNSC08 y DTU10 cuya estadística se calculó con la rutina grdinfo de GMT habiendo previamente excluido el modelo EGM96 o EGM2008 en la zona continental, según correspondiere.

Tabla 2: Estadística descriptiva de las diferencias entre diferentes grillas de anomalías de gravedad derivadas de altimetría. Unidad: [mGal].

\begin{tabular}{c|cccc}
\hline Grilla & Máximo & Mínimo & Media & $\sigma$ \\
SyS18.1-SyS16.1 & 46.45 & -31.66 & -0.240 & 2.19 \\
DNSC08-SyS16.1 & 46.67 & -34.82 & -0.200 & 2.95 \\
DNSC08-SyS18.1 & 28.28 & -24.05 & 0.040 & 1.87 \\
DNSC08-DTU10 & 14.05 & -19.83 & 0.003 & 1.00 \\
\hline
\end{tabular}


Los valores extremos de la diferencia entre las grillas DNSC08 y DTU10 vistos en la Tabla 2 están localizados en una región costera de la provincia de Chubut en las inmediaciones de Camarones y Puerto Piojo.

En las Figuras 3a, 4a y 4b, 5a y 5b se muestra la distribución espacial de las diferencias entre las grillas de anomalía de aire libre calculadas con las diferentes grillas de altimetría satelital SyS18.1, SyS16.1, DNSC08 y DTU10. En la Figura 3b la de las diferencias de anomalías de aire libre calculadas con los modelos geopotenciales EGM2008 y EGM96.

Entre las distintas grillas de altimetría satelital las diferencias son principalmente dadas por la mejora que produce la utilización del modelo geopotencial EGM2008 de mayor resolución que EGM96 como se ve en la Figura 3b, y en el caso de los modelos DNSC08GRA Y V18.1 se deben a los distintos procesamientos principalmente dada la homogeneidad espacial vista en la Figura 4b donde la distribución es casi uniforme y sin tendencia.

Figura 3: Diferencias entre las grillas SyS18.1- SyS16.1(a), izquierda y anomalías de aire libre de EGM2008-EGM96 (b), derecha.

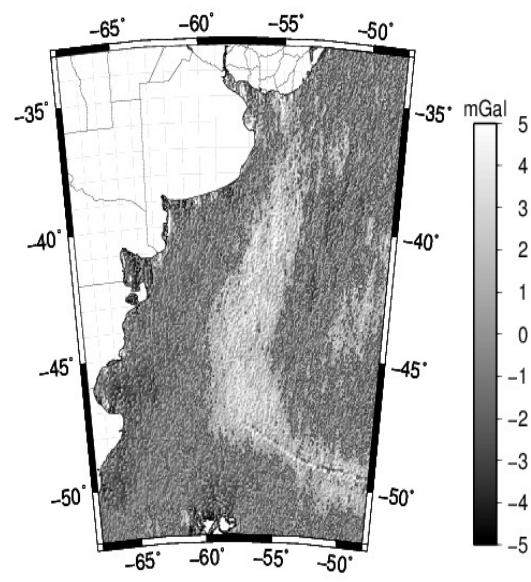

(a)

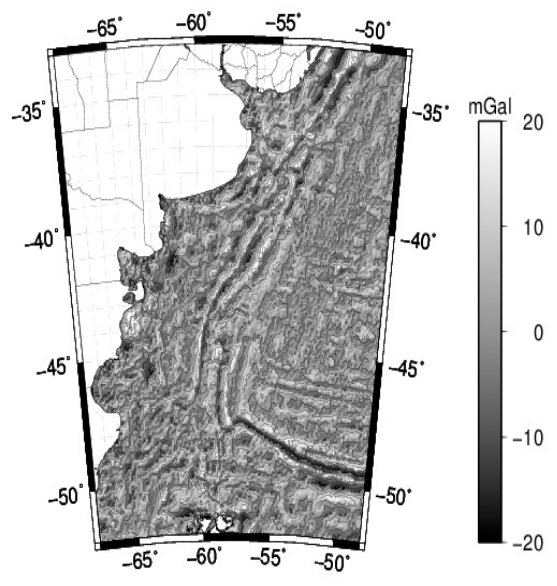

(b)

Es de notar que observando las Figuras 3a, 4a y 4b las estructuras residuales presentes en las diferencias se atenúan cada vez más, sin embargo persisten rasgos estructurales muy marcados en el área del margen continental cizallado y del talud, lo que indica una diferencia más notoria de los modelos al mapear estas zonas .En las Figuras $4 \mathrm{~b}$ y $5 \mathrm{~b}$ se observa una disminución del ruido aleatorio, pero las diferencias estructurales continúan existiendo.

Observando la Figura 5b en forma más detallada, mapeándola con una escala de colores, es posible advertir que la diferencia entre los modelos DNSC08GRA y

Bol. Ciênc. Geod., sec. Artigos, Curitiba, v. 18, nํ1, p.22-39 jan-mar, 2012. 
DTU10GRA muestra un desvío sistemático correlacionado latitudinalmente, a groso modo puede verse como una señal superpuesta de una longitud de onda de aproximadamente $3^{\circ}$ y una amplitud de $\pm 2 \mathrm{mGal}$. El estudio de dicha señal escapa a los objetivos del trabajo, pero sirve para identificar este desvío presente en alguno de los dos modelos o quizás en ambos que no tiene características de una señal con información geofísica sino que es seguramente producto de un artificio matemático.

Figura 4: Diferencias entre las grillas DNSC08-SyS16.1(a), izquierda y DNSC08-SyS18.1 (b), derecha.

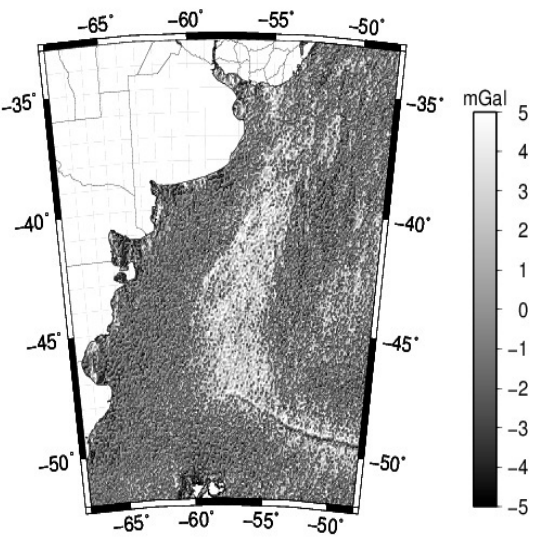

(a)

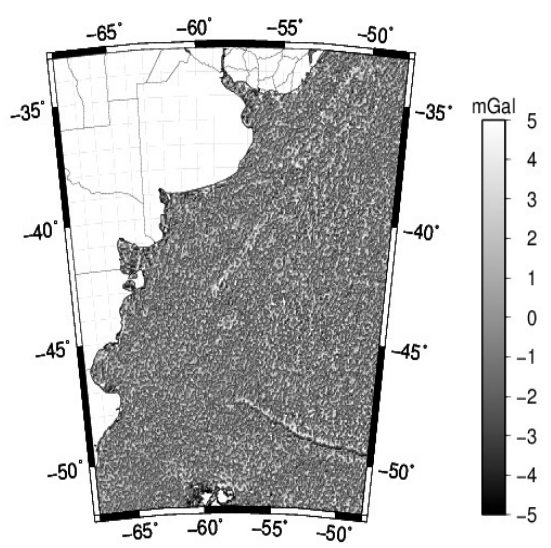

(b)

Figura 5: Diferencias entre las grillas DTU10-SyS18.1(a), izquierda y DNSC08-DTU10 (b), derecha.

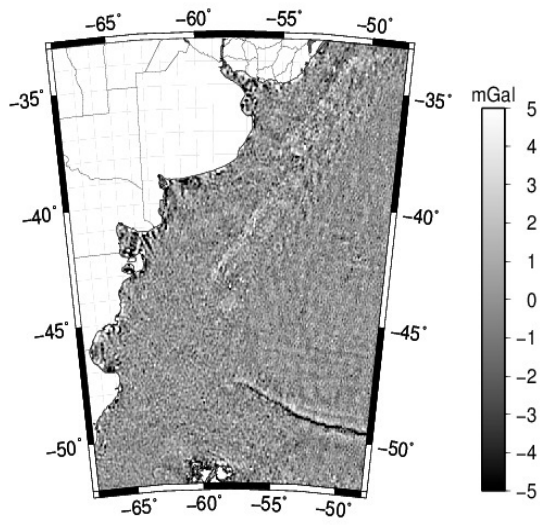

(a)

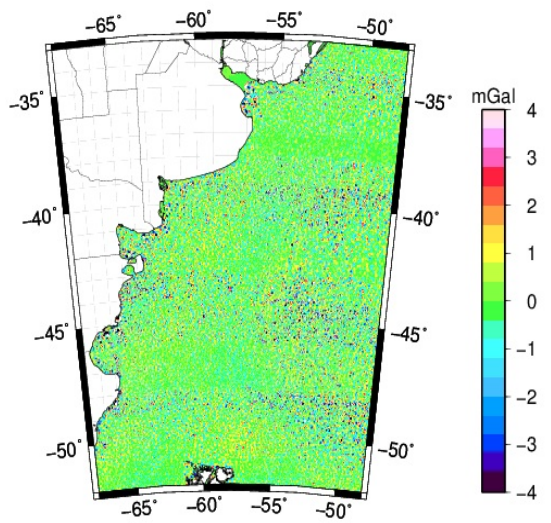

(b) 
4.2 Comparación entre anomalías derivadas de altimetría satelital y anomalías de gravedad marina

Los datos de altimetría fueron interpolados en la localización de las trazas de barco de las diferentes campañas con la rutina grdtrack de GMT con interpolación bilineal. Luego se calcularon las estadísticas de las diferencias entre los valores de las derrotas de barco y los correspondientes interpolados en las grillas para las distintas campañas, las cuales se observan en las Tabla 3 a 6, en las que se muestra la comparación entre las grillas de altimetría y los datos de barco de las campañas BGR87, BGR98-1, BGR98-2 y BGI, respectivamente.

Tabla 3: Estadística de las diferencias entre anomalías de aire libre de la campaña BGR87 y derivadas de altimetría. Unidad: [mGal].

\begin{tabular}{c|cccc}
\hline BGR87 No de puntos :10465 & Máximo & Mínimo & Media & $\sigma$ \\
BGR87-SyS16.1 & 46.36 & -40.75 & 2.72 & 6.14 \\
BGR87-SyS18.1 & 43.27 & -44.20 & 1.23 & 5.73 \\
BGR87-DNSC08 & 43.75 & -42.45 & 1.20 & 5.72 \\
BGR87-DTU10 & 43.26 & -42.75 & 1.10 & 5.62 \\
\hline
\end{tabular}

Tabla 4: Estadística de las diferencias entre anomalías de aire libre de la campaña BGR98-1 y derivadas de altimetría. Unidad: [mGal].

\begin{tabular}{c|cccc}
\hline BGR98-1 No de puntos: 95566 & Máximo & Mínimo & Media & $\sigma$ \\
BGR98-1-SyS16.1 & 23.94 & -16.46 & 0.58 & 4.08 \\
BGR98-1-SyS18.1 & 23.17 & -19.62 & -0.81 & 3.67 \\
BGR98-1-DNSC08 & -20.37 & 20.42 & -0.83 & 3.76 \\
BGR98-1-DTU10 & -20.131 & 20.923 & -0.87 & 3.67 \\
\hline
\end{tabular}

Tabla 5: Estadística de las diferencias entre anomalías de aire libre de la campaña BGR98-2 y derivadas de altimetría. Unidad: [mGal].

\begin{tabular}{c|cccc}
\hline BGR98-2 N de puntos: 76086 & Máximo & Mínimo & Media & $\sigma$ \\
BGR98-2-SyS16.1 & 21.71 & -14.70 & -0.34 & 3.68 \\
BGR98-2-SyS18.1 & 19.53 & -17.37 & -2.45 & 3.21 \\
BGR98-2-DNSC08 & 22.85 & -22.07 & -2.59 & 3.31 \\
BGR98-2-DTU10 & 21.62 & -21.83 & -2.62 & 3.2 \\
\hline
\end{tabular}

Tabla 6: Estadística de las diferencias entre anomalías de aire libre de la campaña BGI y derivadas de altimetría. Unidad: [mGal].

\begin{tabular}{c|cccc}
\hline BGI No de puntos: 7537 & Máximo & Mínimo & Media & $\sigma$ \\
BGI-SyS16.1 & 65.54 & -51.27 & 1.45 & 8.96 \\
BGI-SyS18.1 & 64.42 & -53.28 & 0.91 & 8.93 \\
BGI-DNSC08 & 65.45 & -53.22 & 0.91 & 8.98 \\
BGI-DTU10 & 65.04 & -50.90 & 0.90 & 8.94 \\
\hline
\end{tabular}

Bol. Ciênc. Geod., sec. Artigos, Curitiba, v. 18, nํ1, p.22-39 jan-mar, 2012. 
Para cada juego de comparación se tiene la estadística de las trazas individualizadas, lo que posibilita identificar aquellas que producen las mayores diferencias. Por ejemplo, una línea de la campaña BGI da diferencias del orden de $360 \mathrm{mGal}$ lo que es justificativo para su descarte antes de hacer la estadística de la Tabla 6. Luego se grafican las diferencias en las líneas sobre el mapa de batimetría extraída del modelo DNSC08BAT (ANDERSEN y KNUDSEN, 2008).

Figura 6: Diferencias entre anomalías de aire libre de (BGR87)-(DNSC08), (a) y (BGR98-1)-(DNSC08), (b), sobre mapa de batimetría.

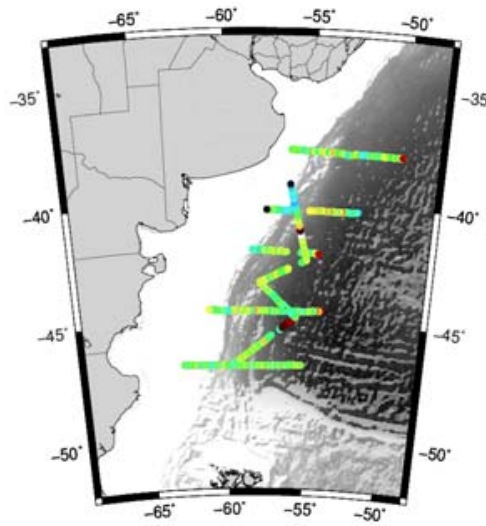

(a)

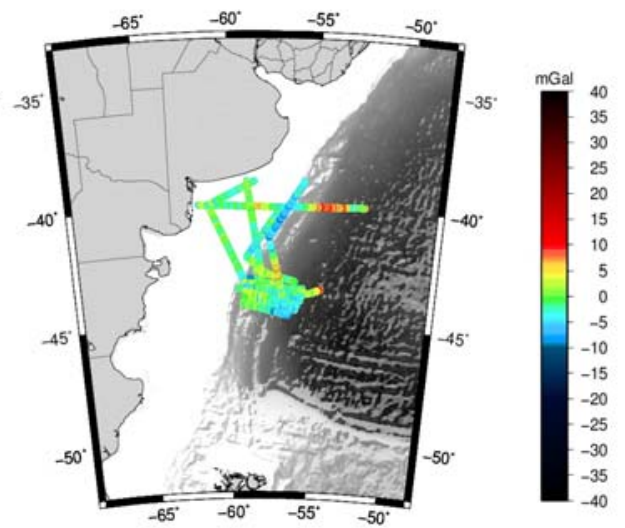

(b)

Figura 7: Diferencias entre anomalías de aire libre de (BGR98-2)-(SyS18.1), (a) y (BGI)-(SyS18.1), (b), sobre mapa de batimetría.

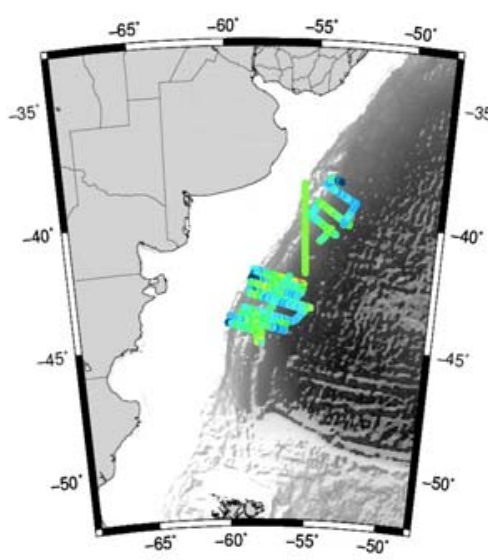

(a)

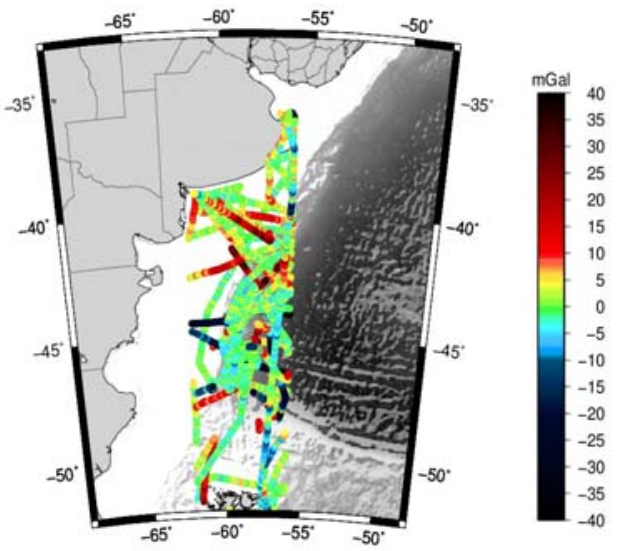

(b)

Bol. Ciênc. Geod., sec. Artigos, Curitiba, v. 18, nº 1, p.22-39, jan-mar, 2012. 
En las Figuras 6 y 7 se observan las diferencias entre las anomalías de gravedad de aire libre de campañas marinas y las anomalías de gravedad derivadas de altimetría satelital. En la Figura 6 BGR87 (a) y BGR98-1 (b) con la grilla DNSC08. En la Figura 7, BGR98-2 (a) y BGI (b) con la grilla SyS18.1.

En las Figuras 6 y 7 se observa que los valores más altos de las diferencias se encuentran al principio o fin de una traza, lo que puede deberse a la influencia del movimiento del barco o a la automatización del sistema de registración y en puntos sobre el borde del talud. Dichos valores a veces son puntuales como el caso de las Figuras 6a y 7a y areales como en el caso de las Figuras $6 \mathrm{~b}$ y $7 \mathrm{~b}$.

Las estadísticas de las diferencias están influenciadas por estos valores puntuales o areales de máxima diferencia. Consecuentemente, se procedió a trabajar en la edición de las trayectorias de los cruceros para detectar y eliminar errores groseros y/o de registración. Esto se hizo con la ayuda de la rutina mapproject de GMT, que calcula la distancia en kilómetros respecto del primer punto del perfil, lo que permite graficar en función de dicha distancia. Esto facilita el análisis individual, encontrar y eliminar valores erróneos manualmente.

En las Figuras $6 \mathrm{~b}$ y $7 \mathrm{~b}$ además los errores están asociados al hecho de ser mediciones muy próximas a la costa o en aguas someras donde los modelos de altimetría satelital continúan teniendo problemas dado que las técnicas de retracking no mejoran en este sentido (ANDERSEN et al., 2010) y la precisión de las anomalías de gravedad marina derivada de la altimetría no es la misma que en regiones de océano abierto. La razón de estas discrepancias en zonas costeras se debe a 1) que la topografía y la rugosidad del mar contaminan el frente de onda de los altímetros, 2) las mediciones altimétricas no pueden corregirse adecuadamente al existir inexactitud de las correcciones geofísicas de mareas en aguas someras y retardo atmosférico, 3) formas inapropiadas o imposibles de limpiar los datos de gravedad marina (análisis de cross-over y correcciones asociadas), 4) el hecho de no poder tener uniformidad en los datos en regiones costeras que es una necesidad al usar FFT (gaps en las costas generan efectos de borde), (DENG et al.,2002).

El proceso de edición requiere una inspección visual para eliminar errores groseros y una minuciosa búsqueda de regiones ruidosas en las que debe evaluarse su eliminación con el compromiso de evitar la pérdida innecesaria de datos. Requiere entonces un tiempo dado que es manual y no es conveniente convertirlo en un proceso automatizado.

Se hizo nuevamente el cálculo de la comparación entre las anomalías derivadas de altimetría y las de las campañas editadas, y los resultados se muestran en las Tabla 7 a 10, en donde se eliminaron líneas cortas y muy ruidosas.

Para hacer más explícita la información contenida en las Tablas estadísticas 3 a 6 y 7 a 10 se grafican histogramas los que se muestran en las Figuras 8 a 11 . 
Tabla 7: Estadística de las diferencias entre anomalías de aire libre de la campaña BGR87 editada y derivadas de altimetría. Unidad: [mGal].

\begin{tabular}{c|cccc}
\hline BGR87 No de puntos: 10114 & Máximo & Mínimo & Media & $\sigma$ \\
BGR87-SyS16.1 & 13.43 & -10.04 & 1.88 & 3.53 \\
BGR87-SyS18.1 & 14.29 & -10.12 & 0.42 & 3.02 \\
BGR87-DNSC08 & 15.63 & -9.64 & 0.40 & 3.03 \\
BGR87-DTU10 & 12.95 & -10.18 & 0.30 & 2.87 \\
\hline
\end{tabular}

Tabla 8: Estadística de las diferencias entre anomalías de aire libre de la campaña BGR98-1 editada y derivadas de altimetría. Unidad: [mGal].

\begin{tabular}{c|cccc}
\hline BGR98-1 N de puntos: & Máximo & Mínimo & Media & $\sigma$ \\
95418 & & & & \\
BGR98-1-SyS16.1 & 23.86 & -12.05 & 0.57 & 4.06 \\
BGR98-1-SyS18.1 & 23.17 & -12.24 & -0.81 & 3.66 \\
BGR98-1-DNSC08 & 20.42 & -12.24 & -0.85 & 3.74 \\
BGR98-1-DTU10 & 20.92 & -12.74 & -0.88 & 3.65 \\
\hline
\end{tabular}

Tabla 9: Estadística de las diferencias entre anomalías de aire libre de la campaña BGR98-2 editada y derivadas de altimetría. Unidad: [mGal].

\begin{tabular}{c|cccc}
\multicolumn{5}{c}{ BGR98-2 editada y derivadas de altimetría. Unidad: [mGal]. } \\
\hline BGR98-2 No de puntos: & Máximo & Mínimo & Media & $\sigma$ \\
75870 & & & & \\
BGR98-2-SyS16.1 & 16.29 & -14.70 & -0.34 & 3.66 \\
BGR98-2-SyS18.1 & 13.79 & -17.37 & -2.46 & 3.18 \\
BGR98-2-DNSC08 & 16.08 & -22.07 & -2.59 & 3.28 \\
BGR98-2-DTU10 & 14.88 & -21.83. & -2.62 & 3.17 \\
\hline
\end{tabular}

Tabla 10: Estadística de las diferencias entre anomalías de aire libre de la campaña BGI editada y derivadas de altimetría Unidad: [mGal].

\begin{tabular}{c|cccc}
\hline BGI N de puntos: 7080 & Máximo & Mínimo & Media & $\sigma$ \\
BGI-SyS16.1 & 33.40 & -32.84 & 2.16 & 6.52 \\
BGI-SyS18.1 & 35.06 & -34.27 & 1.68 & 6.40 \\
BGI-DNSC08 & 36.14 & -35.05 & 1.67 & 6.44 \\
BGI-DTU10 & 36.22 & -34.85 & 1.65 & 6.40 \\
\hline
\end{tabular}


Figura 8: Histograma de las diferencias BGR87-DNSC08 crudo (a), izquierda y editado (b), derecha.

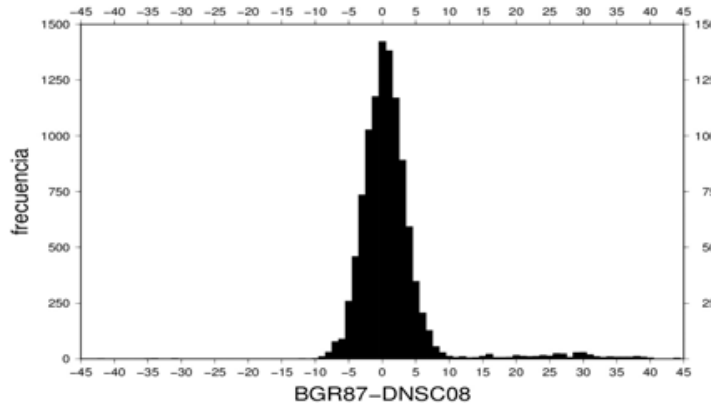

(a)

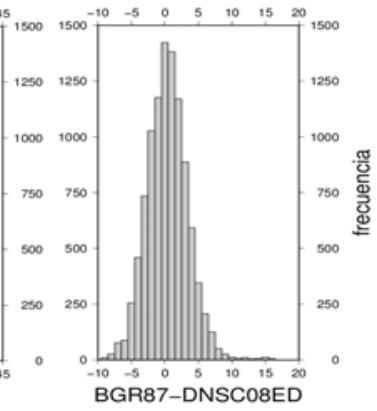

(b)

Figura 9: Histograma de las diferencias BGR87-SyS16.1 cruda (a), izquierda y editada (b), derecha.

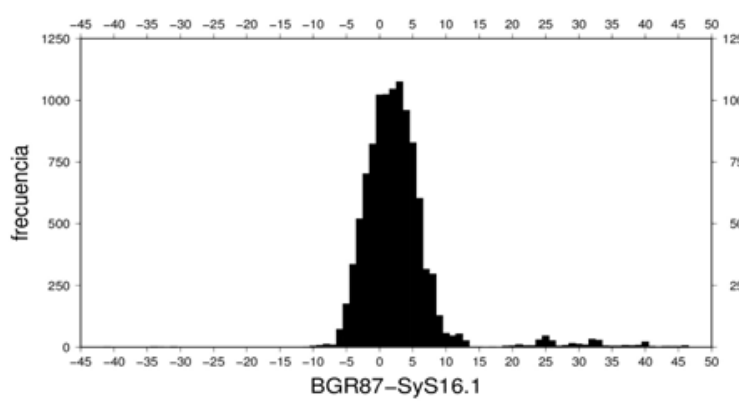

(a)

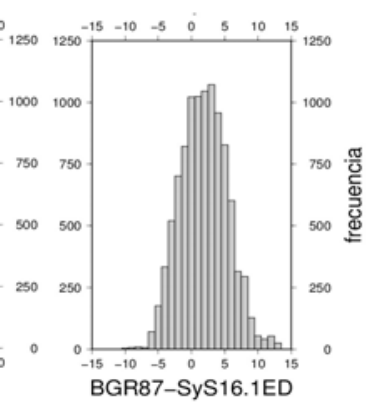

(b)

Figura 10: Histograma de las diferencias BGI-SyS16.1 cruda(a), izquierda y editada (b), derecha.

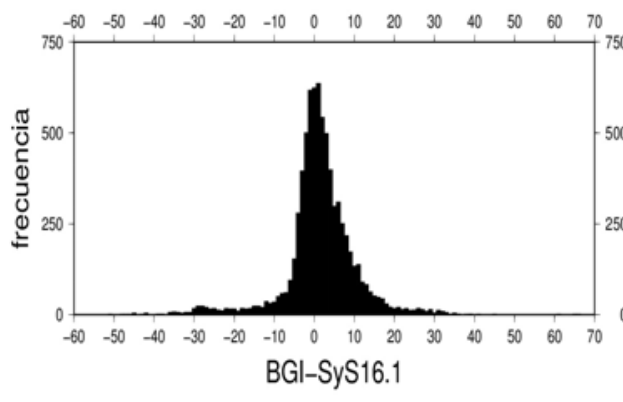

(a)

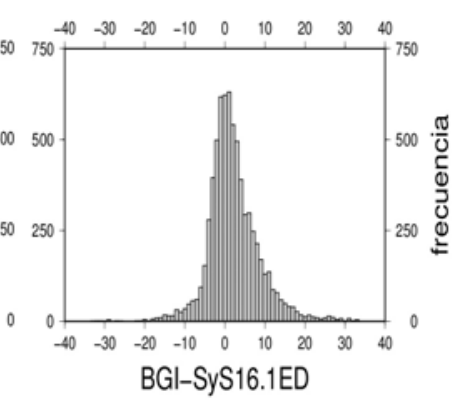

(b)

Bol. Ciênc. Geod., sec. Artigos, Curitiba, v. 18, nํ1, p.22-39 jan-mar, 2012. 
Figura 11: Histograma de las diferencias BGI-SyS18.1 cruda (a), izquierda y editada (b), derecha.

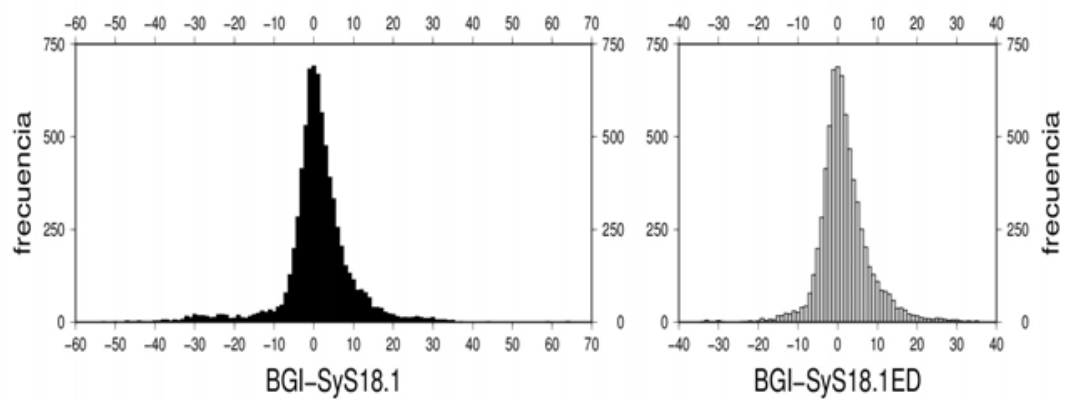

(a)

(b)

En las Figuras 8 a 11 la Figura de la izquierda (a) representa el estado crudo de las diferencias, en las que sólo se han eliminado derrotas con diferencias groseras, y en la figura de la derecha (b) el resultado luego de la edición de las trazas y su posterior comparación con la altimetría satelital. En las Figura 8 y 9 se muestra las diferencias entre la campaña marina BGR87 y las grillas de altimetría satelital DNSC08 y SyS16.1, respectivamente. En las Figuras 10 y 11 se muestra las diferencias entre la campaña marina BGI y las grillas de altimetría satelital SyS16.1 y SyS18.1, respectivamente.

\section{CONCLUSIONES}

Las mejoras introducidas en los modelos DTU10GRA, DNSC08GRA y V18.1 hacen que sean los más adecuados para su uso. Las ventajas de éstos modelos frente al modelo V16.1 se deben, entre otras, al uso de un mejor modelo geopotencial durante el procesamiento y principalmente, a los distintos procesamientos utilizados para su realización.

El nuevo modelo DTU10GRA mostró tener un mejor acuerdo al disminuir la media y desviación estándar de las diferencias entre campaña marina-altimetría satelital respecto a DNSC08GRA de casi todos los conjuntos de datos. Sin embargo existe un desvío sistemático correlacionado latitudinalmente en la diferencia entre ambos modelos.

En la comparación con anomalías de gravedad marina las diferencias más grandes son debidas a: 1) las influencias del movimiento de rotación del barco en el inicio o finalización de una traza, 2) la distribución de los datos marinos en la región del margen continental argentino en la zona del talud continental dado que la resolución de los modelos DNSC08GRA, DTU10GRA y V18.1 es buena en dicha región, 3) a los errores inherentes a la altimetría satelital en regiones costeras o de aguas poco profundas.

Bol. Ciênc. Geod., sec. Artigos, Curitiba, v. 18, nํ1, p.22-39, jan-mar, 2012. 
La utilización de datos de barco de diferentes fuentes requiere un análisis de la coherencia o consistencia de los mismos a través de en una serie de pasos de depuración y posterior ajuste.

En primera instancia se eliminaron líneas de datos de barco con errores muy groseros, de la comparación directa con los datos derivados de altimetría satelital. Luego se siguió con una serie de pasos tendientes a mejorar la consistencia interna de las campañas, en los que el primer paso es la edición manual de los datos, paso que tratará de hacerse más exhaustivamente. Siguieron luego los pasos necesarios para practicar un ajuste por cruces o nivelación, proceso que aun no se ha completado y se recomienda continuar.

\section{TRABAJOS FUTUROS}

La coherencia interna de cada campaña se mide a través del análisis de cruces (cross-overs), para lo que se buscan aquellas líneas que más puntos de cruce tengan por campaña para su reajuste, calculándose las diferencias de los valores de gravedad en esos puntos. Se propone realizar un ajuste polinómico por mínimos cuadrados que modele la variación del valor de anomalía de gravedad en el cruce en función de la distancia al primer punto de la línea seleccionada, con lo cual se mejorará la coherencia interna de la campaña. Posteriormente se practicaría el análisis de cruces entre campañas para mejorar la coherencia externa o cruzada. En cada paso se tratará de minimizar las diferencias con las anomalías de gravedad derivadas de altimetría satelital. Cuando haya finalizado todo este proceso la grilla que mejor ajusta en la región del margen continental argentino podrá entonces combinarse con anomalías de gravedad marina en una base de datos unificada, dando mayor homogeneidad a los datos medidos desde plataformas a bordo de barcos para su utilización en diferentes aplicaciones.

\section{AGRADECIMIENTOS}

Agradecemos la disponibilidad de los datos de las campañas marinas de Bureau Gravimétrique Internacional (BGI) y de las campañas BGR87, BGR98-1 y BGR98-2 realizadas por el Federal Institute for Geosciences and Natural Resources, obtenidas para su utilización en proyectos científicos en el margen continental argentino por gestiones de M. Ghidella.

\section{REFERENCIAS BIBLIOGRÁFICAS}

ANDERSEN, O. B.; KNUDSEN, P. Global marine gravity field from the ERS-1 and Geosat geodetic mission altimetry, Journal of Geophysical Research, v. 103, n. C4, p. 8129-8137, 1998.

ANDERSEN, O.B.; KNUDSEN, P. The DNSC08BAT bathymetry developed from satellite altimetry, EGU meeting, Vienna, Austria, 2008.

ANDERSEN, O.B.; KNUDSEN, P.; BERRY, P. The DNSC08 global marine gravity field from retraced satellite altimetry, Journal of Geodesy, v. 84, n. 3, p. 191-199, 2009.

Bol. Ciênc. Geod., sec. Artigos, Curitiba, v. 18, nํ1, p.22-39 jan-mar, 2012. 
ANDERSEN, O.B.; KNUDSEN, P.; BERRY, P.; SMITH, R. Recent developments in high-resolution global altimetric gravity field modeling, The Leading Edge, v. 29, n.5, p. 540-545, 2010.

ANDERSEN, O. B. The DTU10 Gravity field and Mean sea surface, Second international symposium of the gravity field of the Earth (IGFS2), Fairbanks, Alaska, 2010.

DENG, X.; FEATHERSTONE, W.E.; Hwang, C.; Shum, C.K. Improved Coastal Marine Gravity Anomalies at the Taiwan Strait from Altimeter Waveform Retracking, In: Proceeding of the international Workshop on Satellite Altimetry for Geodesy, Geophysics and Oceanography, Wuhan, 8-13 sept. 2002.

DENKER, H; ROLAND, M. Compilation and evaluation of a consistent marine gravity data set surrounding Europe, Proc IUGG General Assembly, Sapporo, Japan, Springer, Berlin, 2003.

DNSC08GRA disponible en <http://www.space.dtu/>, 2009.

DTU10GRA disponible en <http://www.space.dtu/>, 2012.

FEATHERSTONE, W. E. Comparison of different satellite altimeter-derived gravity anomaly grids with ship-borne gravity data around Australia, in: Tziavos, I.N. (ed) gravity and Geoid 2002, Department of Surveying and Geodesy, Aristotle University of Thessaloniki, p 326-331, 2003.

LEMOINE, F. G.; KENYON, S. C.; FACTIM, J. K.; TRIMMER, R. G.; PAVLIS, N. K.; CHINN, D. S.; COX, C. M.; KLOSKO, S. M.; LUTHCKE, S. B.; TORRENCE, M. H.; WANG, Y. M.; WILLIAMSON, R. G. ; PAVLIS, E. C.; RAPP, H. ; OLSON, T. R. The Development of the Joint NASA GSFC and the National Imagery and Mapping Agency (NIMA) Geopotential Model EGM96; NASA Technical Paper NASA/TP1998206861, Goddard Space Flight Center, Greenbelt, USA, 1998.

PAVLIS, N. K.; HOLMES, S.A.; KENYON, S. C.; FACTOR, J. K. An earth Gravitational Model to degree 2160 :EGM2008, presented at the 2008 general assembly of the European Geosciences Union, Vienna, Austria, April 13-18, (pdf file whit the original power point presentation), 2008.

SANDWELL, D. T.; SMITH, W. H. F. Marine gravity anomaly from Geosat and ERS 1 satellite altimetry, Journal of Geophysical Research, v. 102, n. B5, p. 10.039-10.054, 1997 .

SANDWELL, D. T.; SMITH, W. H. F. Global marine gravity from retracked Geosat and ERS 1 altimetry: Ridge segmentation versus spreading rate, Journal of Geophysical Research, v. 114, n. B01411, doi: 10.1029/2008JB006008, 2009.

V16.1 y V18.1 disponibles en <ftp://topex.ucsd.edu/pub/>, 2009.

WESSEL, P.; SMITH, W. H. F. The Generic Mapping Tools Technical Reference and Cookbook, Version 4.2.0, p. 155, 2007.

(Recebido em janeiro de 2012. Aceito em março de 2012). 